\title{
Os Livros Filosóficos InACABAdos de PeSsoa. Problemas e Critérios para a PublicaÇão dos EsCRITOS FILOSÓfiCOS DE PESSOA
}

\author{
Nuno Filipe Ribeiro \\ (Doutorando na Universidade Nova de Lisboa) \\ "Tenho feito filosofias em segredo que nenhum Kant escreveu" \\ Álvaro de Campos, Tabacaria
}

Num escrito autobiográfico de Pessoa relativo à sua escrita, lemos:

Nenhum dos meus escritos foi concluído; sempre se interpuseram novos pensamentos, associações de ideias extraordinárias, impossíveis de excluir, com o infinito como limite. Não consigo evitar a aversão que tem o meu pensamento pelo acto de acabar seja o que for. [...]

O meu carácter é tal que eu detesto o princípio e o fim das coisas, pois são pontos definidos. ${ }^{1}$

Este texto toca num aspecto fundamental com o qual todo o editor de Pessoa se confronta. No espólio de Pessoa encontramos uma multiplicidade de esboços, esquemas e fragmentos destinados a livros que Pessoa pretendia futuramente escrever. Mas o facto é que esses livros, cujo princípio se verifica entre os escritos de Pessoa, nunca, ou quase nunca, foram concluídos. Com efeito, se excluirmos o Livro de Odes de Ricardo Reis - que é mais uma colecção de poemas do que um livro estruturado -,

${ }^{1}$ «My writings were none of them finished; new thoughts intruded ever, extraordinary, inexcusable associations of ideas bearing infinity for term. I cannot prevent my thoughts hatred of finish[ing]; [...] My character of mind is such that I hate the beginnings and the ends of things, for they are definite points.»: Fernando Pessoa, Escritos Autobiográficos, Automáticos e de Reflexão Pessoal, Lisboa, Assírio \& Alvim, 2003, p. 100. Seguimos a tradução presente na edição.

Philosophica, 38, Lisboa, 2011, pp. 165-174 
Pessoa apenas publicou um livro no decurso da sua vida. Esse livro é a Mensagem. Pessoa publicou diversos poemas e ensaios em revistas literárias que criou ou com as quais colaborou, como nos mostra Fernando Cabral Martins nas suas edições intituladas Crítica $^{2}$ e Ficções de Interlúdio. ${ }^{3}$ No entanto, nenhum desses poemas e ensaios constitui um livro por si só e, na maioria dos casos, são apenas partes ou capítulos de livros mais extensos projectados por Pessoa. Assim, no espólio de Pessoa existe uma multiplicidade de projectos para futuros livros. Entre os livros do espólio de Pessoa cujo princípio se constata, mas que não chegaram a ser concluídos, encontram-se os livros filosóficos de Pessoa.

O espólio de Pessoa, catalogado na Biblioteca Nacional de Lisboa [BNP] sob a designação de «E3» [Espólio 3], encontra-se dividido por envelopes e compreende mais de vinte e sete mil documentos. Cada envelope está classificado com um número, uma designação e contém um número variável de documentos. Entre os diversos envelopes do espólio Pessoa existem quatorze envelopes filosóficos com 1428 documentos. Existem cinco envelopes $\left(15^{1}, 15^{2}, 15^{3}, 15^{4}\right.$ e $\left.15^{5}\right)$ com a designação «Filosofia», um (15A) classificado como «Filosofia-Metafísica», quatro $\left(15 \mathrm{~B}^{1}, 15 \mathrm{~B}^{2}, 15 \mathrm{~B}^{3}\right.$ e $\left.15 \mathrm{~B}^{4}\right)$ designados como «Filosofia-Psicologia»e, finalmente, quatro $(22,23,24$ e 25) intitulados «Textos Filosóficos».

No que diz respeito ao estado da arte da publicação dos escritos filosóficos de Pessoa, existe uma edição em dois volumes intitulada Textos Filosóficos de Fernando Pessoa, publicada por António de Pina Coelho na Editorial Ática ${ }^{4}$ e que foi recentemente reeditada pela mesma editora ${ }^{5}$. Mas esta edição tem vários problemas. Antes de mais, contém apenas uma pequena selecção de textos filosóficos, sobretudo os mais fáceis de transcrever, e a selecção e transcrição dos textos está circunscrita a quatro envelopes filosóficos $(22,23,24$ e 25), representando somente uma pequena percentagem dos documentos presentes nesses envelopes. $\mathrm{O}$ segundo problema é que esta edição não possui um critério crítico para a selecção e organização dos textos. Os textos são reunidos de acordo com conceitos temáticos que não correspondem nem a um princípio temático de organização estabelecido por Pessoa, nem a um projecto do espólio concebido por Pessoa para reunir um grupo de textos filosóficos.

\footnotetext{
2 See.: Fernando Pessoa, Crítica, Lisboa, Assírio \& Alvim, 2000.

${ }^{3}$ See: Fernando Pessoa, Ficções do Interlúdio, Assírio \& Alvim, 1998.

${ }^{4}$ A referência da edição é: Fernando Pessoa, Textos Filosóficos de Fernando Pessoa (estabelecidos e prefaciados por António Pina Coelho), Lisboa, Ática, 1968.

${ }^{5}$ A referência da edição é: Fernando Pessoa, Textos Filosóficos de Fernando Pessoa (estabelecidos e prefaciados por António Pina Coelho), Lisboa, Editorial Nova Ática, 2006.
} 
Para além destes dois volumes, encontramos no livro Pessoa Inédi$t o^{6}$, organizado por Teresa Rita Lopes, um capítulo com 22 páginas, contendo uma selecção de textos filosóficos feita por José Gil. Esta selecção é mais criteriosa do que a realizada por António de Pina Coelho, mas é feita apenas com a intenção de introduzir o grande público à presença de pensamento filosófico no espólio de Pessoa. Finalmente, existem também referências esporádicas aos textos filosóficos em algumas edições dos escritos de Pessoa tais como Escritos sobre Génio e Loucura editado por Jerónimo Pizarro, na qual se faz alusão a um projecto destinado a um livro filosófico em inglês com o título On Free-Will. ${ }^{7}$ Mas esta referência, tal como outras referências esporádicas, não é, nem pretende ser, uma apresentação completa dos textos filosóficos, pois a sua finalidade não é a edição de todos os escritos filosóficos de Pessoa. Tendo em consideração todos os aspectos referidos relativos ao estado da arte da publicação dos escritos filosóficos de Pessoa, devem, então, ser estabelecidos os critérios para a publicação desse grupo de textos. Mas antes disso, é necessário definir aquilo que deve ser compreendido como escrito filosófico no contexto da publicação dos textos de Pessoa.

O primeiro problema com o qual o editor de Pessoa se depara no confronto com os textos presentes no espólio de Pessoa é o problema da autoria. Os textos de Pessoa são escritos sob vários nomes. Pessoa produz os seus escritos com a intenção de os atribuir a e publicar sob o nome de diferentes personalidades. Assim o editor que queira publicar Pessoa deve ter essa pluralidade de atribuição autoral em consideração. Contudo, existem também textos sem qualquer atribuição autoral, isto é, textos que Pessoa cria para incluir em obras mais vastas, mas que não têm nenhuma referência autoral explícita ou implícita. Este é o caso da maioria dos escritos filosóficos de Pessoa. Assim, o que é que pode ser considerado como um texto que deve ser publicado como escrito filosófico? Todos os textos que fazem referência a conceitos filosóficos, independentemente da sua atribuição ou apenas uma parte dos escritos filosóficos? Se o critério é a publicação de uma parte apenas, qual é a parte que deve ser considerada como publicável? A tradição editorial de Pessoa - e por tradição editorial entendo a Edição Crítica coordenada por Ivo Castro e a edição das Obras de Fernando Pessoa publicada pela Assírio e Alvim - segue o princípio segundo o qual todas as obras atribuídas a uma determinada

${ }^{6}$ AAVV, Pessoa Inédito (coordenação Teresa Rita Lopes), Lisboa, Livros Horizonte, 1993: ver especificamente as páginas 106-109 (2.13. "Onde o poeta se manifesta animado pela filosofia”) e 397-420 («8. Um poeta animado pela Filosofia»).

${ }^{7}$ Ver: Fernando Pessoa, Escritos sobre Génio e Loucura, Lisboa, Imprensa Nacional Casa da Moeda, 2006, p. 235. 
personalidade devem ser editadas sob o nome da personalidade à qual são atribuídas. Os poemas e a prosa de Ricardo Reis, por exemplo, devem ser publicados sob o nome de Ricardo Reis, os poemas e a prosa de Álvaro de Campos sob o nome de Álvaro de Campos e assim por diante. Existem excepções a este princípio. Mas isso acontece apenas em casos específicos, com uma intenção editorial explícita, como no caso dos volumes Crítica e Fiç̧ões do Interlúdio editados por Fernando Cabral Martins com a intenção de estabelecer a cronologia dos textos publicados por Pessoa ao longo da sua vida. Assim, tendo todos estes aspectos em consideração, os textos que devem ser publicados sob a designação de escritos filosóficos são todos os textos presentes no espólio de Pessoa e que não contenham nenhuma referência autoral explícita ou implícita, o que corresponde à maioria dos escritos filosóficos. Isso não significa que alguns dos textos atribuídos por Pessoa a uma determinada personalidade não possam ser publicados em apêndice a alguns dos escritos filosóficos. Nos casos em que exista uma afinidade temática entre um texto filosófico com atribuição autoral e um texto sem atribuição autoral, o texto com atribuição autoral poderá ser incluído em apêndice ao outro texto. Essa é a prática de alguns dos volumes da Edição Crítica de Pessoa. No entanto, para além da afinidade temática não existe uma regra geral para determinar se se deve ou não incluir um texto com atribuição autoral em apêndice. Deve decidir-se caso a caso.

O segundo problema com o qual o editor de Pessoa se depara, no que respeita aos escritos filosóficos, é o problema da topografia dos textos. Como já foi referido, o espólio Pessoa contém quatorze envelopes filosóficos. Mas se, por um lado, existem quatorze envelopes de índole filosófica, por outro lado, o facto é que existem escritos filosóficos que não estão nos quatorze envelopes filosóficos e os envelopes filosóficos contêm, de igual modo, escritos que não são filosóficos. Num envelope com a designação "Páginas de Estética e de Teoria e Crítica Literárias" [envelope 19] encontramos, por exemplo, um texto sobre a filosofia de Nietzsche intitulado «Friedrich Nietzsche» $[\mathrm{BNP} / \mathrm{E} 3-19-99]^{8}$ e num envelope com a designação "Textos Filosóficos" [envelope 23] existe um texto com o título «De Profundis» [BNP/E3-23-66], publicado por António de Pina Coelho em Textos Filosóficos de Fernando Pessoa, ${ }^{9}$ mas que, no entanto, é uma tradução de um excerto de De Profundis de Óscar Wilde.

\footnotetext{
${ }^{8}$ Este texto encontra-se publicado em: Fernando Pessoa, Páginas de Estética e de Teoria e Crítica Literárias, Lisboa, Ática, 1994, pp. 333-334.

${ }^{9}$ Cf. Fernando Pessoa, Textos Filosóficos de Fernando Pessoa (estabelecidos e prefaciados por António Pina Coelho), Volume I, Lisboa, Ática, 1994. p. 227 e 228.
} 
Os envelopes com a designação «Filosofia-Psicologia» (envelopes $15 \mathrm{~B}^{1}$ a $15 \mathrm{~B}^{4}$ ) são também um caso especial no espólio de Pessoa. Embora estes envelopes sejam classificados sob a designação de «filosofia», eles contêm escritos que Pessoa classifica sob o conceito de «Microsofia: a Ciência do Diminuto» [«Microsphy: the Science of the Minute»] [BNP/E3, 24-120v], que é um termo criado por Pessoa para designar as pequenas ciências, tais como a frenologia, a fisionomia, assim como outras pequenas ciências relacionadas com a psiquiatria e não um grupo de textos que deva ser editado sob a designação de "Filosofia". Assim, a edição dos escritos filosóficos de Pessoa não deve nem estar circunscrita aos envelopes filosóficos nem conter todo o material presente nesses envelopes.

Tendo chegado a uma definição daquilo que deve ser publicado numa edição dos escritos filosóficos, é necessário enumerar as classes ou grupos de textos e documentos filosóficos que existem no espólio de Pessoa. Os escritos filosóficos podem ser classificado em cinco grupos.

O primeiro grupo de textos filosóficos compreende os livros filosóficos inacabados. Existem dois tipos de livros filosóficos inacabados. O primeiro tipo engloba os escritos de Pessoa concebidos como parte de projectos. No espólio de Pessoa existem diversos documentos com projectos feitos pelo próprio Pessoa. Esses projectos encontram-se divididos em diversos tópicos, correspondentes a vários capítulos ou linhas de pensamento que Pessoa pretendia desenvolver em livros por si projectados. Muitos desses tópicos correspondem a diversas páginas que existem no espólio de Pessoa. Com efeito, a designação dos tópicos presente em muitos dos projectos corresponde ao título e às indicações de uma multiplicidade de páginas espalhadas ao longo do espólio de Pessoa. Entre os documentos com projectos de Pessoa, encontramos os projectos de livros filosóficos. O seguinte texto redigido por Pessoa em inglês e os seus diversos tópicos, que correspondem a um projecto destinado a um livro filosófico intitulado Categories, constituem um exemplo de um projecto filosófico de Pessoa:

Book I - Theory of Categories.

[Book] II - Theory following on, and depending on the Categories.

[Book] III - Theory of the Absolute.

\section{Subdivisions:}

Book I-1. Category of Being. Considerations.

2. Category of Extension. Considerations.

3. Category of Relation. Consideration.

4. $<$ Conclusion $>$ Critique of Pure Reason.

5. Conclusion. 
Book II - 1. Relation of Objects to Categories.

2. Proof of the Inexistence of the External World.

$\left[\mathrm{BNP} / \mathrm{E} 3,15^{3}-3\right]$

$\mathrm{O}$ segundo tipo de livros filosóficos inacabados engloba aqueles escritos que não correspondem a nenhum projecto, mas cujo argumento pode ser reconstruído quer de acordo com as indicações presentes nos documentos, quer segundo afinidades temáticas que entre esses documentos existam. Um exemplo deste tipo de livro filosófico inacabado é um texto escrito em inglês intitulado Rationalism, que se encontra espalhado ao longo do espólio de Pessoa (como a maioria dos escritos filosóficos), que não corresponde a nenhum projecto, mas que tem inúmeras indicações que nos permitem reconstruir o argumento do livro. Assim, existe no envelope $15^{2}$ uma série de nove documentos dactiloscritos com a designação «Rationalism» $\left[\mathrm{BNP} / \mathrm{E} 3,15^{2}-62^{\mathrm{r}}\right.$ a $\left.70^{\mathrm{r}}\right]$ e, por exemplo, no envelope $15^{3}$ encontra-se um grupo de nove textos manuscritos com o título «Rationalism» ou com a abreviação «Rat. ${ }^{\mathrm{m}}{ }_{\text {» }}\left[\mathrm{BNP} / \mathrm{E} 3,15^{3}-37^{\mathrm{r}}\right.$ a $\left.45^{\mathrm{r}}\right]$.

$\mathrm{O}$ segundo grupo de escritos filosóficos corresponde aos ensaios e pequenas produções. Existem vários géneros de ensaios com extensões diversas e que discutem o mais variado tipo de assuntos, autores e movimentos filosóficos. Por exemplo, no envelope $15^{1}$ existe uma série de dois documentos que correspondem à primeira parte de um ensaio inglês inacabado intitulado Essay on Heraclitus [BNP/E3, $15^{1}-52^{\mathrm{r}}$ a $53^{\mathrm{v}}$ ]. No envelope $15^{5}$ existe também uma série de onze páginas que correspondem a um ensaio relativamente maior sobre a ciência e o conhecimento [BNP/E3, 155-1-11]. Existem mais de 30 ensaios filosóficos e pequenas produções no espólio de Pessoa e estes documentos são apenas dois exemplos dessa variedade de textos.

O terceiro grupo de textos corresponde às notas de leitura de Pessoa. Pessoa tinha o hábito de tomar notas das suas leituras, não só de literatura mas também de filosofia, entre outras áreas do conhecimento. Isto acontecia a maior parte das vezes porque Pessoa lia, principalmente na Biblioteca Nacional de Lisboa (como se pode verificar nos seus diários), livros que não eram seus, mas cuja referência queria manter. A publicação das suas notas é de crucial importância, não só para traçar as várias influências filosóficas na obra de Pessoa, mas também porque muitas dessas notas estão na origem de alguns dos seus escritos filosóficos. Assim, podemos distinguir dois tipos de notas: primeiro, as notas acríticas, que compreendem todas aquelas notas que têm meras indicações ou citações de livros filosóficos que Pessoa leu; segundo, as notas críticas, que, para além das referências e citações de livros, englobam também algumas considerações de Pessoa sobre aquilo que leu. Um exemplo de uma nota 
acrítica é um documento intitulado «Critique of Pure Reason - Introduction» [BNP/E3, $15^{5}-76$ e 77], que, tal como o texto indica, é um resumo da "Introdução" da Crítica da Razão Pura de Kant. No envelope 22 existe um exemplo de uma nota de leitura crítica, como se pode verificar no seguinte texto escrito por Pessoa em inglês sobre a definição aristotélica de metafísica:

\section{Notes 1 .}

Aristotle defines metaphysics: (1) science of first principles and of first causes and (2) science of being as being [qua being] $\square$

Also defined "the science of the absolute."

Metaphysics contains 3 great theories: 1) Theory of knowledge, 2) theory of being, 3) theory of the first principle of knowledge and of being.

Critique. It contains great theories indeed. These are: 1) Theory of knowledge, 2) $\square$

[BNP/E3, 22-73]

O quarto grupo de escritos filosóficos é composto pelos diálogos filosóficos. Pessoa não pretendeu expressar as suas ideias filosóficas apenas através de livros e ensaios, mas planeou também criar diversos diálogos filosóficos e trabalhar temas filosóficos através da discussão entre as personagens desses diálogos. No envelope 24 existe um documento precisamente intitulado «Diálogos filosóficos» [BNP/E3, 24 - 96a].

Finalmente, o último grupo de textos filosóficos corresponde às páginas filosóficas autónomas. Este tipo de textos engloba todos aqueles escritos que lidam com a filosofia ou com assuntos filosóficos, mas que não correspondem a nenhum dos outros grupos filosóficos, nem têm qualquer relação material directa com os outros escritos filosóficos. Um exemplo deste grupo de textos pode ser observado num texto com o título «Ethics» [BNP/E3, 154-21].

A divisão dos textos filosóficos em cinco grupos é a base para o estabelecimento de critérios para uma edição dos escritos filosóficos de Pessoa. Se os escritos filosóficos fossem ordenados de acordo com critérios estritamente cronológicos, o resultado seria o mesmo que se encontra no espólio de Pessoa, isto é, um conjunto de textos caótico e sem qualquer ligação. Assim, uma edição ordenada de acordo com critérios estritamente cronológicos apresentaria exactamente aquilo que Pessoa diz no excerto citado no início deste texto: pensamentos que se interpõem uns diante dos outros e associações de ideias sem conexão. No entanto, isto não significa que o editor não deva ter a preocupação com a cronologia dos escritos. A edição dos escritos filosóficos de Pessoa deverá ser seguida de um índice que permita ao leitor e estudioso de Pessoa seguir a cro- 
nologia dos escritos. Para além do índice com a cronologia dos textos, o editor deverá também estabelecer um índice com a ordem numérica dos escritos no espólio de Pessoa, para possibilitar ao leitor o confronto entre o texto estabelecido na edição e o documento original.

Por outro lado, a transcrição dos textos deverá obedecer a princípios critico-genéticos. Os textos deverão ser apresentados da forma mais limpa possível e transcritos de acordo com a intenção final do autor. Isso significa que quando houver mais de uma variante disponível para uma palavra ou frase, o editor deverá sempre escolher a última variante deixada pelo autor. Para além disso, o estabelecimento do texto deverá ser sempre seguido de um aparato genético que proporcione todas as informações relativas ao documento, tais como o material, o tipo de documento e as lições excluídas do texto pelo editor, como as variantes alternativas e as palavras e frases riscadas. O estabelecimento de um aparato genético é de crucial importância por três razões: primeira, porque o estudo do material poderá proporcionar pistas para o estabelecimento de um índice com a cronologia; segundo, porque a apresentação das variantes eliminadas e das palavras ou frases riscadas permitirão que o estudioso de Pessoa siga o processo criativo do autor; finamente, porque, com a degradação do material, o aparato genético permitirá reter informações que, de outro modo, não estariam disponíveis para o estudioso de Pessoa.

No que diz respeito aos sinais utilizados para a transcrição dos textos, o editor deverá usar a chave dos símbolos empregue pela edição crítica de Fernando Pessoa. Esses símbolos são:

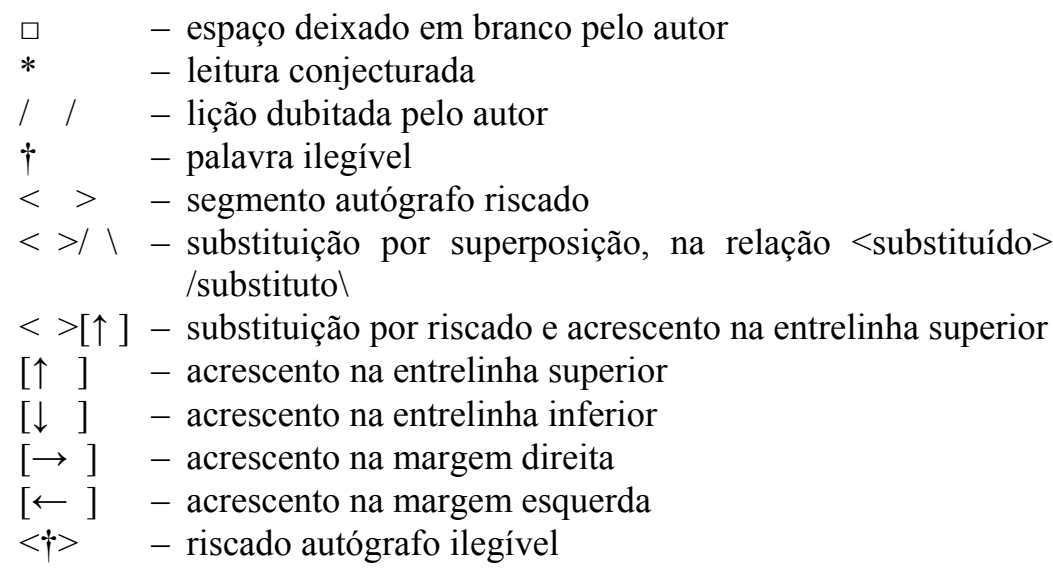

Para além destes sinais, o editor deverá servir-se de dois outros símbolos: 
Primeiro: [ ] - para indiciar todas as intervenções do editor no texto (este sinal já é empregue pela Edição Crítica de Pessoa, ainda que isso não seja explicitamente declarado).

Segundo: $\leftrightarrow-$ para indicar a presença de duas palavras separadas que se encontram, acidentalmente ou não, escritas conjuntamente no decurso do texto (isto acontece, a maior parte das vezes, nos textos dactiloscritos de Pessoa).

Assim, tendo em consideração estes princípios, a edição dos escritos filosóficos de Pessoa deverá estar dividida em cinco partes, correspondentes aos cinco grupos de escritos filosóficos acima descritos e mencionados: primeiro, os livros filosóficos inacabados, organizados de acordo com os projectos deixados por Pessoa ou com as indicações presentes nos seus escritos; segundo, os ensaios filosóficos e pequenas produções; terceiro, as páginas filosóficas autónomas; quarto, os diálogos filosóficos; quinto, as notas de leitura filosóficas. Dentro de cada uma dessas partes, o editor deverá tentar organizar o texto (ou grupo de textos, no caso se escritos mais extensos) desde o primeiro na ordem de produção e criação literária de Pessoa até ao mais tardio. Por exemplo, os livros filosóficos inacabados deverão ser ordenados desde o primeiro grupo de escritos destinados a um livro filosófico até ao grupo de textos mais tardio. Mas a ordem dos escritos dentro de cada grupo de textos concebidos para um projecto deverá ser aquela que Pessoa deixou indicada no documento. Assim, cada documento será agrupado de acordo com o destino que Pessoa deixou indicado nele e com os diversos tópicos dos projectos.

Deste modo, o trabalho de edição dos escritos filosóficos de Pessoa deverá ter três etapas. A primeira etapa será a investigação da biblioteca filosófica de Pessoa. Por biblioteca filosófica de Pessoa deve compreender-se não só os livros que materialmente se encontram na «Casa Fernando Pessoa», mas também aqueles livros que se encontram identificados nas listas de leitura de Pessoa. A investigação da biblioteca de Pessoa permitirá que o editor distinga, de uma forma mais clara, os escritos filosóficos de Pessoa e as suas notas de leitura filosóficas. Também proporcionará material para notas críticas sobre os escritos filosóficos, pois Pessoa tinha o hábito de comentar os seus livros, com uma vasta marginalia, enquanto os lia. Por exemplo, entre os escritos filosóficos de Pessoa existem inúmeras páginas de um projecto para um ensaio intitulado Essay on Free-Will, que se sabe, a partir da consulta da biblioteca de Pessoa, que é uma resposta a e inspirado pelo livro Essai sur le Libre Arbitre, ${ }^{10}$ que é

10 Ver: Jerónimo Pizarro, Patricio Ferrari and Antonio Cardiello, A Biblioteca Particular de Fernando Pessoa/Pessoa's Private Library, Lisboa, D. Quixote, 2010, p. 95 
uma tradução francesa de Über die Freiheit des Willens de Schopenhauer. A segunda etapa será a pesquisa do espólio de Pessoa. Nesta pesquisa, o editor deverá identificar claramente todos os escritos filosóficos espalhados ao longo do espólio. Na terceira etapa o editor deverá seleccionar, transcrever e organizar os textos de acordo com os projectos e as classes de documentos filosóficos acima indicados.

De tudo o que foi dito deve concluir-se que os escritos filosóficos de Pessoa são um campo inexplorado, contendo uma multiplicidade de regiões ainda por descobrir. De facto, num dos seus escritos filosóficos, Pessoa diz:

Milhares de teorias, grotescas, extraordinárias, profundas, sobre o mundo, sobre o homem, sobre todos os problemas que pertencem à metafísica atravessaram o meu espírito. Tive em mim milhares de filosofias das quais - como se fossem reais - nem mesmo duas concordariam. ${ }^{11}$

Assim, se é verdade aquilo que Pessoa diz neste excerto, a edição dos escritos filosóficos de Pessoa será um modo de mostrar uma das facetas - talvez a menos conhecida - da produção deste autor e de tornar mais produtivos os estudos da obra de Pessoa.

\title{
RESUMO
}

O presente artigo debate o alcance filosófico da obra de Fernando Pessoa. Com efeito, no espólio de Pessoa encontramos inúmeros projectos destinados a futuros livros, ensaios, pequenas produções e diálogos filosóficos. Assim, partindo de uma análise dos documentos, em grande parte inéditos, do espólio de Pessoa, pretende demonstrar-se que a dimensão filosófica da obra deste autor se estende muito além dos ecos de leituras filosóficas presentes na sua poesia e nas suas ficções.

\begin{abstract}
This article debates the philosophical reach of Pessoa's writings. As a matter of fact, Pessoa's Archive contains several projects for philosophical books, essays, small productions and dialogues. Thus, this article tries to demonstrate, through the analysis of the unpublished documents, that the philosophical dimension of this author's work is not circumscribed to the philosophical references present in the poetry and fictions of Fernando Pessoa.

11 «Thousands of theories, grotesque, extraordinary, profound, on the world, on man, on all problems that pertain to metaphysics have passed through my mind. I have had in me thousands of philosophies not any two of which - as if they were real agreed.»: AAVV, Pessoa Inédito, p. 402.
\end{abstract}

